\title{
Phenotyping, functional characterization, and developmental changes in canine intestinal intraepithelial lymphocytes
}

\author{
Nicole Luckschander ${ }^{1,2}$, Nadia S. Pfammatter ${ }^{1,2}$, Daniel Sidler ${ }^{1}$, \\ Sabine JAKob ${ }^{1}$, Iwan A. Burgener ${ }^{2}$, Peter F. Moore ${ }^{3}$, Andreas Zurbriggen ${ }^{2}$, \\ Nadia CORAZzA ${ }^{1 *}$, Thomas BRUNNER ${ }^{1 *}$ \\ ${ }^{1}$ Division of Immunopathology, Institute of Pathology, University of Bern, Murtenstrasse 31, \\ 3010 Bern, Switzerland \\ ${ }^{2}$ Department of Clinical Veterinary Medicine, Vetsuisse Faculty, University of Bern, Länggasstrasse 128, \\ 3012 Bern, Switzerland \\ ${ }^{3}$ Department of Pathology, Microbiology and Immunology, School of Veterinary Medicine, \\ University of California, Rm. 1126 Haring Hall, Davis, CA 95616, USA
}

(Received 18 March 2009; accepted 23 July 2009)

\begin{abstract}
Little is currently known about the lymphocyte populations in the normal and diseased canine gut. The aim of this study was thus the phenotypical and functional characterization of canine intestinal intraepithelial lymphocytes (IEL). IEL were isolated from full-thickness biopsies of 15 adult Swiss Beagle dogs (mean age $8.2 \pm 2.8$ years) and compared to mesenteric lymph node cells. The phenotypical characterization by multi-parameter flow cytometry revealed that canine IEL differ substantially from lymph node $\mathrm{T}$ cells, and consist of various unconventional lymphocyte subsets, unique to mucosal surfaces. These include $\gamma \delta$ T cells, and $\mathrm{CD} 4^{-} \mathrm{CD} 8^{-}$and $\mathrm{CD} 8 \alpha \alpha^{+} \mathrm{T}$ cells. IEL populations in adult dogs were also compared to those isolated from neonatal Beagle dogs. Analysis revealed a high frequency of undifferentiated $\mathrm{CD} 4^{-} \mathrm{CD} 8^{-} \mathrm{T}$ cells in newborn dogs whereas mature $\mathrm{CD} 4^{+}$and $\mathrm{CD} 8^{+} \mathrm{T}$ cells predominate in adult dogs, indicating maturation of the intestinal immune system during development. As IEL in other species are thought to exhibit regulatory functions, we investigated the role of IEL on the activation-induced proliferation of lymph node T cells. While IEL alone did not show activation-induced proliferation, they significantly inhibited the proliferation of activated lymph node $\mathrm{T}$ cells in a cell number-dependent manner. These findings are the first to demonstrate that canine intestinal IEL have an immunoregulatory phenotype, which may contribute to the maintenance of intestinal immune homeostasis and may, therefore, be lost in canine chronic enteropathies.
\end{abstract}

dog / intestine / intraepithelial lymphocyte

\section{INTRODUCTION}

The gastrointestinal tract plays the key role in the uptake of fluids and nutrients, and at

\footnotetext{
* Corresponding authors: nadia.corazza@pathology. unibe.ch, tbrunner@pathology.unibe.ch
}

the same time it forms the main protective barrier between the sterile environment of the body and the outside world [10]. To protect the body from invading pathogens, the intestinal mucosa has developed one of the largest and most complex organ-associated immune systems [19]. Under normal physiological conditions, the 
intestinal immune system is tightly regulated to avoid overshooting reactions. Loss of this regulatory control mechanism in inflammatory bowel disease (IBD) leads to perpetuating inflammatory and infiltrative responses. In both humans and dogs, the development of intestinal mucosal inflammatory disorders, such as IBD, is observed with increasing frequency [19]. Despite the high incidence of IBD in dogs, the mechanistic events leading to these disease entities are poorly understood. Although several lines of evidence point towards an important role of intestinal lymphocytes in the development of canine IBD, the precise patho-mechanisms are currently not understood.

In this study, we characterized intestinal intraepithelial lymphocytes (IEL), a developmentally and functionally unique lymphocyte compartment, strategically located in the intestinal epithelial layer as a first line of defense. IEL are readily accessible by simple dissociation from the epithelial cells, and changes in the composition of the IEL compartment likely reflect ongoing immunological responses in the intestinal mucosa. The phenotypical composition of IEL may therefore represent a sensitive surrogate marker for pathological changes in the intestine, such as those occurring during IBD. A limited number of previous studies have investigated the distribution and quantification of IEL in the canine intestine. Most of these were descriptive studies using immunohistochemistry and did not include the simultaneous analysis of multiple lymphocyte markers $[5-8,14]$. In addition, intestinal tissue samples used in previous studies were collected from a variety of different breeds, which may have lead to a high inter-sample variability due to breed-related differences in the composition of IEL. The goal of the present study was to perform an in-depth phenotypical and functional characterization of small and large intestinal IEL in a single breed (the Beagle) in order to establish an unbiased baseline for future studies on the role and fate of intestinal $\mathrm{T}$ cells in the development of canine enteropathies. Developmental changes in the IEL compartments were analyzed by comparing lymphocyte subsets in adult dogs to those of neonatal dogs with an average age of 1 day. Phenotypical changes were analyzed by multi-parameter flow cytometry. Functional parameters analyzed consisted of activation-induced proliferation and cytokine expression profiles. Our data describe hitherto unrecognized lymphocyte subsets in the canine intestinal epithelium and their developmental changes from newborn to adult dogs. Furthermore, we provide evidence that IEL may have a regulatory role in the healthy dog as they potently inhibit activation-induced proliferation of lymph node $\mathrm{T}$ cells.

\section{MATERIALS AND METHODS}

\subsection{Animals}

Tissue samples were collected from adult $(n=15)$ and neonatal $(n=6)$ healthy Swiss Beagle dogs. All dogs were provided from either the Novartis Centre de Recherche Santé Animale (St. Aubin, Switzerland) or the RCC Ltd Laboratory Animal Service (Füllinsdorf, Switzerland). The adult dogs (mean age $8.2 \pm 2.8$ years, 8 male, 7 female) were clinically healthy animals, which were euthanized for reasons unrelated to any gastrointestinal abnormality. The neonatal puppies (average age 1 day, 5 male, 1 female) were culled from a laboratory animal breeding facility due to excessive numbers. Immediately after euthanasia by barbiturate injection, full thickness sections from the intestine (ascending duodenum, mid-jejunum and mid-colon) and mesenteric lymph nodes were collected and further processed for cell isolation or immunohistochemistry. All animal experiments were conducted according to the guidelines of the Animal Experimentation Committee of the Canton of Bern, Switzerland.

\subsection{Antibodies}

All monoclonal antibodies used for flow cytometry were specific for canine leukocyte antigens. Antibodies against canine CD4 (clone YKIX302.9, $\mathrm{PE}$ conjugate), CD3 (clone CA17.2A12, FITC conjugate), $\mathrm{CD} 8 \beta$ (clone $\mathrm{CA} 15.4 \mathrm{G} 2), \mathrm{CD} 8 \alpha$ (clone YCATE55.9, FITC conjugate) and CD45 (clone YKIX 716.13, biotin conjugate) were purchased from Serotec (Morphosys AbD GmbH, Düsseldorf, Germany). Monoclonal antibodies against canine TCR $\alpha \beta$ (clone CA15.8G7), TCR $\gamma \delta$ (clone CA20.8H1) were generated as previously described [7] and purified on protein-G columns. Antibodies against TCR $\alpha \beta$ and TCR $\gamma \delta$ were labeled with biotin 
according to standard protocols. Unlabeled antibodies were detected using R-PE-conjugated goat antimouse IgG $\mathrm{F}(\mathrm{ab}) 2$ fragment (BD Becton Dickinson, Allschwil, Switzerland), biotinylated antibodies with Cy5-conjugated streptavidin (THP Medical Products, Vienna, Austria). Immunohistochemical detection of IEL was performed with rabbit anti-human CD3 antibody (Neomarkers, Fremont, CA, USA).

\subsection{Immunohistochemistry}

Intestinal samples were fixed in $4 \%$ paraformaldehyde in PBS and embedded in paraffin. Five $\mu \mathrm{m} \mathrm{sec-}$ tions were dewaxed and pretreated by boiling for

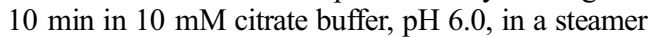
to retrieve antigen. Endogenous peroxidase activity was blocked with peroxidase blocking solution (S2001, Dako, Zug, Switzerland) and unspecific binding was blocked with $5 \%$ goat serum, $0.5 \%$ casein and $0.1 \%$ sodium azide in TBS (Ab dilution buffer). Tissue sections were then stained with either rabbit anti-human CD3 antibody (1:200) in Ab dilution buffer or a control antibody for $1 \mathrm{~h}$ at room temperature (RT) in a moist chamber. Purified rabbit immunoglobulin was used at $1 \mu \mathrm{g} / \mathrm{mL}$ as a staining control. The specificity of the anti-human CD3 antibody was previously tested on sorted $\mathrm{CD} 4^{+}$canine T cells. After 3 washes in TBS, sections were stained with biotinylated goat anti-rabbit Ig antiserum (1:100, Dako Cytomation) for $1 \mathrm{~h}$ at RT. Sections were then washed and incubated with a streptavidin-biotin/ horse radish peroxidase conjugate $(1: 200$, Dako) for $40 \mathrm{~min}$. Finally, sections were washed and developed with a peroxidase substrate solution $\left(0.2 \mathrm{mg} / \mathrm{mL} 3,3^{\prime}\right.$ diaminobenzidine tetrahydrochloride $\mathrm{DAB}, 0.012 \%$ $\mathrm{H}_{2} \mathrm{O}_{2}, 20 \mathrm{mM}$ citric acid monohydrate, $100 \mathrm{mM}$ imidazole, $100 \mathrm{mM} \mathrm{NaCl}, \mathrm{pH} \mathrm{7.0)}$ for $7 \mathrm{~min}$ in the dark and counterstained with hematoxylin. Sections were then dehydrated with an increasing concentration of ethanol and finally mounted.

\subsection{IEL isolation}

Full thickness tissue samples from the small bowel (ascending duodenum and mid-jejunum) and the large bowel (mid-colon) were used for IEL isolations. Tissue samples were examined for the presence of Peyer's patches, which were excluded from the isolation protocol. Intestinal tissue samples were then cut into pieces of $1 \times 1 \mathrm{~cm}$ containing the entire mucosa (villi and crypts). Tissue samples from duodenum and mid-jejunum were pooled for IEL isolation. The IEL were isolated by previously published protocols, which were adapted to canine lymphocytes $[2,15]$. In brief, IEL were dissociated from intestinal tissue by gently stirring tissue pieces in $\mathrm{Ca}^{2+}$ - and $\mathrm{Mg}^{2+}$-free HBSS containing $2 \%$ horse serum, $1 \mathrm{mM}$ DTT, and $0.5 \mathrm{mM}$ EDTA for $2 \times 30 \mathrm{~min}$ at $37{ }^{\circ} \mathrm{C}$. Isolated cells were sequentially passed through a $70 \mu \mathrm{m}$ and a $40 \mu \mathrm{m}$ pore nylon mesh cell strainer (Becton Dickinson, San Jose, CA, USA). The IEL were then separated from enterocytes by a $40 \% / 70 \%$ Percoll (Parmacia Biotech, Uppsala, Sweden) gradient centrifugation (15 min, $900 \times g$, RT). Cells were collected from the interphase and washed twice in PBS, $2 \%$ horse serum. Cell numbers and viability were estimated using a hemocytometer and trypan blue dye exclusion. IEL preparations generally contained between $15 \%$ and $70 \%(57 \pm 21 \%) \mathrm{CD} 45^{+}$leukocytes.

\subsection{Isolation of lymph node lymphocytes}

Mesenteric lymph nodes were mechanically disrupted, passed through a $40 \mu \mathrm{m}$ pore nylon mesh cell strainer and washed once with PBS, 2\% horse serum. Cell numbers and viability were evaluated using a hemocytometer and trypan blue dye exclusion. Mesenteric lymph node preparations generally contained between $70 \%$ and $90 \%(84.3 \pm 8.5 \%)$ $\mathrm{CD} 45^{+}$leucocytes.

\subsection{Flow cytometry}

Mesenteric lymph node lymphocytes and IEL from small and large bowel were stained with differentially labeled antibodies as indicated in the results. For double stainings, 100000 viable cells were incubated with primary fluorescent dye-labeled antibodies (anti-CD3-FITC, 1:100 dilution; anti-CD4-PE, 1:1 000 dilution; CD8 $\alpha$-FITC, $1: 20$ dilution) or biotinylated antibodies (anti-CD45-biotin, 1:200 dilution; anti-TCR $\alpha \beta, 1: 200$ dilution; anti-TCR $\gamma \delta, 1: 200$ dilution) in a 96-well V-bottom plate for $15 \mathrm{~min}$ at $4{ }^{\circ} \mathrm{C}$ in the dark. After two washes with $\mathrm{PBS} / 2 \%$ horse serum, cells were resuspended in $100 \mu \mathrm{L}$ PBS $/ 2 \%$ horse serum containing Cy5-conjugated streptavidin and incubated for $15 \mathrm{~min}$ at $4{ }^{\circ} \mathrm{C}$ in the dark. Cells were then washed again, fixed and analyzed by flow cytometry. For the simultaneous detection of $\mathrm{CD} 8 \alpha$, $\mathrm{CD} 8 \beta$ and TCR, triple stainings were used as follows. Viable cells were first incubated with rat anti-CD8 $\alpha$ FITC (1:20 dilution) and mouse anti-CD8 $\beta$ (1:100 dilution) antibodies. After two washes with $\mathrm{PBS} / 2 \%$ horse serum, cells were resuspended in $100 \mu \mathrm{L}$ $\mathrm{PBS} / 2 \%$ horse serum containing goat anti-mousePE (1:300 dilution) and incubated for $15 \mathrm{~min}$ at $4{ }^{\circ} \mathrm{C}$ in the dark. Cells were washed and unsaturated goat anti-mouse antibody was blocked by incubation 
with $1 \mathrm{mg} / \mathrm{mL}$ mouse $\mathrm{IgG}$ at $4{ }^{\circ} \mathrm{C}$ in the dark for $10 \mathrm{~min}$. After an additional wash, biotinylated mouse anti-canine TCR $\alpha \beta$ (1:200 dilution) or TCR $\gamma \delta$ (1:200 dilution) antibodies were added for $15 \mathrm{~min}$. Cells were then washed, and biotin was detected with Cy5-conjugated streptavidin (1:500 dilution). After a final wash, all samples were fixed in $1 \%$ phosphatebuffered formaldehyde and analyzed by flow cytometry on a FACScan (BD Biosciences). Data were analyzed using flow cytometry software (FlowJo, Tree Star Inc., Ashland, OR, USA). For analysis, cells were normalized to $\mathrm{CD} 45^{+}$cells. Data were reported as the mean $\pm \mathrm{SD}$. Statistical analysis was done using the Mann-Whitney $U$-test, as indicated in the text.

\subsection{Proliferation assay}

Small intestinal IEL and lymphocytes from mesenteric lymph nodes, isolated from 8 adult healthy Beagle dogs using the protocol described above, were either directly used for proliferation assays $(n=5)$ or stored overnight in cell culture medium (Iscove's Modified Dulbeccos's Medium, IMDM), $2 \mathrm{mM}$ L-alanyl glutamine, $5 \% \mathrm{FCS}, 50 \mu \mathrm{g} / \mathrm{mL}$ gentamycine) at $4{ }^{\circ} \mathrm{C}$ for further purification by cell sorting $(n=3)$. IEL were separated from contaminating epithelial cells based on forward/side scatter properties (lymphocyte gate) using a FACStar cell sorter (BD Biosciences). The purity of the sorted IEL was confirmed by $\mathrm{CD} 3$ staining and usually contained $>99 \%$ $\mathrm{CD}^{+}$cells. IEL and lymph node lymphocytes were plated at a density of $1 \times 10^{5}$ cells/well in 96-well U-bottom microtiter plates in complete medium in triplicates. In some experiments, lymph node lymphocytes were co-cultured with decreasing numbers of IEL, starting at a 1:1 ratio (unsorted cells) and a 1:3 ratio (sorted cells) respectively, followed by subsequent three-fold dilutions. Non-specific effects of contaminating epithelial cells in unpurified IEL preparations on lymph node $\mathrm{T}$ cell proliferation were assessed by sorting epithelial cells on a cell sorter and identical co-culture with lymph node cells. Cells were then stimulated with medium control or PMA $(50 \mathrm{ng} /$ $\mathrm{mL})$ and ionomycin $(500 \mathrm{ng} / \mathrm{mL})$ as a polyclonal T cell stimulus. Cultures were incubated for $24 \mathrm{~h}$ at $37{ }^{\circ} \mathrm{C}$ in $5 \% \mathrm{CO}_{2}$ and then pulsed with $1 \mu \mathrm{Ci} /$ well ${ }^{3} \mathrm{H}$-thymidine for $6 \mathrm{~h}$. Cells were harvested on glass fiber filters and ${ }^{3} \mathrm{H}$-thymidine incorporation was measured on a beta counter (TopCount, Perkin Elmer).

\subsection{Real time PCR}

Duodenal IEL and lymphocytes from the mesenteric lymph nodes of 8 adult healthy Beagle dogs were isolated and sorted by flow cytometry as described above. Cells were then stimulated for $6 \mathrm{~h}$ with PMA $(50 \mathrm{ng} / \mathrm{mL})$ and ionomycin (500 ng/ $\mathrm{mL}$ ), harvested and lyzed in TRI reagent (Sigma Aldrich). Total RNA was isolated and cDNA was generated using a high-capacity cDNA Kit (Applied Biosystems, Foster City, CA, USA). Real-time PCR was performed on an Applied Biosystems 7500 real-time PCR instrument using SYBR green and canine-specific primer sets (Quantitech, Qiagen, Hilden, Germany) for GAPDH, IFN $\gamma$, TGF $\beta$ and IL-10. Data were normalized to the expression levels of the house-keeping gene GAPDH.

\section{RESULTS}

\subsection{Immunohistochemical detection of IEL in the canine small and large intestine}

Tissue sections from the small and large canine intestine, stained with an anti-CD3 antibody, demonstrated numerous $\mathrm{CD}^{+}$cells in the epithelium and the lamina proria (Figs. 1B and 1C). Control reagents did not reveal any positive staining (Fig. 1A). Higher magnification showed that $\mathrm{CD}^{+}$cells are embedded in-between the epithelial cells luminal to the basal membrane, with a frequency of approximately $1-2$ every 10 epithelial cells.

\subsection{Canine intestinal IEL consist predominantly of $\mathrm{CD}^{+} 5^{+} \mathrm{CD3}^{+} \mathrm{T}$ cells}

Analysis of isolated intestinal IEL by flow cytometry demonstrated a clearly distinguishable population in the lymphocyte gate. Staining of these cells for the common leukocyte marker CD45 and the $\mathrm{T}$ cell marker CD3 revealed that $>95 \%$ of the cells within the lymphocyte gate were $\mathrm{CD} 45^{+}$and $\mathrm{CD}^{+}$(Fig. 2).

\subsection{Canine intestinal IEL consist of conventional and unconventional $T$ cell subsets}

The $\mathrm{T}$ cell compartment in the mesenteric lymph node of adult Beagle dogs was found to be composed predominantly of $\mathrm{CD}^{+}$and $\mathrm{CD}^{+} \mathrm{T}$ cells, as is known to be the case in peripheral lymphoid organs. In marked contrast, 


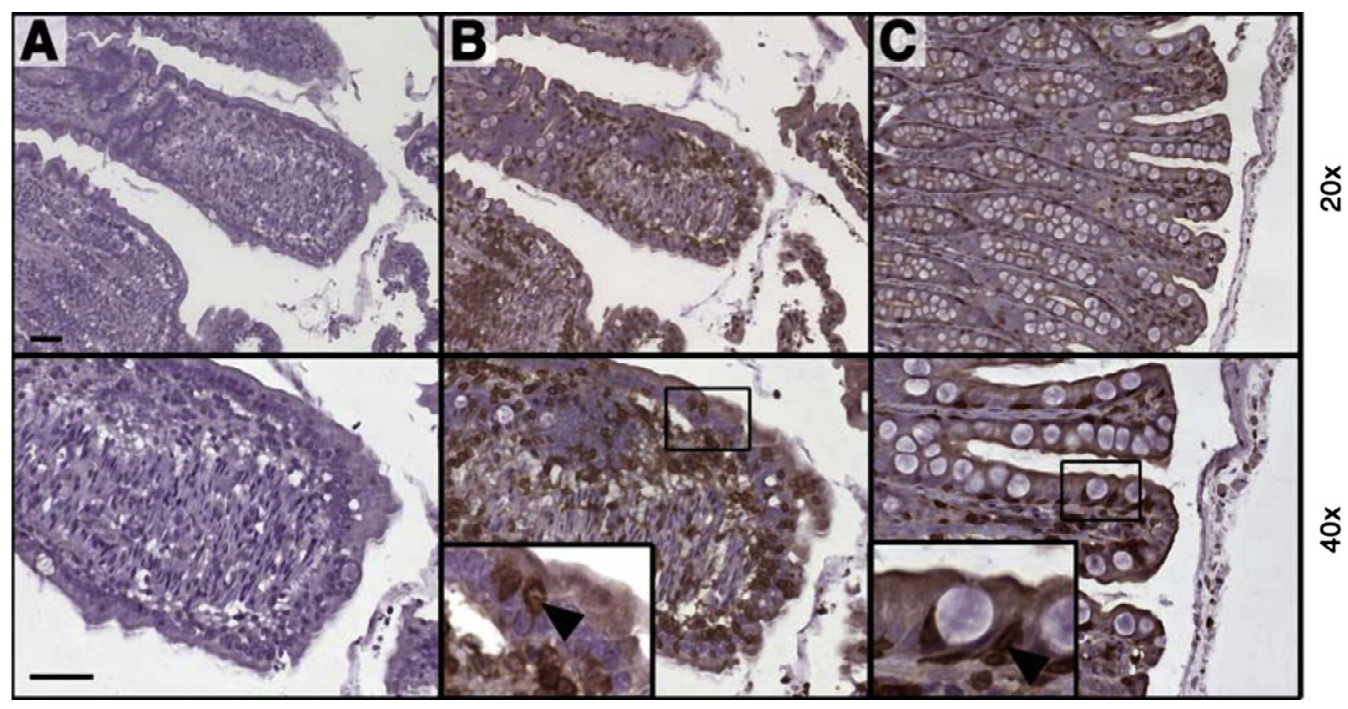

Figure 1. Immunohistochemical detection of $\mathrm{CD}^{+}$cells in the small and the large canine intestine. Paraffin sections of canine small and large intestine were stained with control rabbit IgG (A) or anti-CD3 antibody $(B, C)$. Lower $(20 \times)$ and higher magnifications $(40 \times)$ of representative stainings of small $(A, B)$ and large intestine (C) are shown. Inserts show enlargements of the epithelial layer with IEL (arrow). Bars indicate $50 \mu \mathrm{m}$.

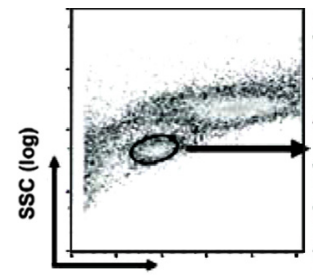

FSC (lin)

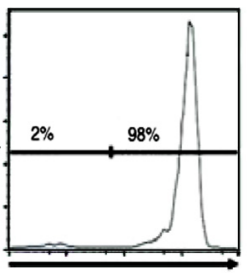

$\operatorname{CD} 45(\log )$

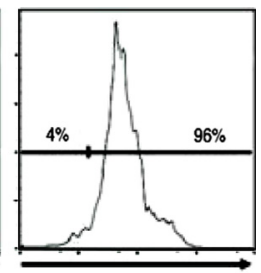

CD3 $(\log )$

Figure 2. Characterization of canine intestinal IEL. Flow cytometric analysis of IEL isolated from the small bowel of an adult Beagle dog, stained with anti-canine CD45 and anti-canine CD3. The distinct forward/ side scatter properties of IEL and their staining for CD45 and CD3 in a typical isolation are shown.

only few $\mathrm{CD}^{+}$cells $(<15 \%)$ were found in the intestinal epithelium, with slightly higher numbers in the small bowel (Fig. 3A). Most $\mathrm{T}$ cells in the peripheral immune system are known to be TCR $\alpha \beta^{+}$with only very few unconventional $\mathrm{TCR} \gamma \delta^{+}$cells. We also found a dominant population of TCR $\alpha \beta^{+} \mathrm{T}$ cells in the intestinal epithelial layer and in the mesenteric lymph nodes. However, a substantial proportion of unconventional $\mathrm{TCR} \gamma \delta^{+} \mathrm{T}$ cells (small bowel $22.9 \pm 6.3 \%$; large bowel
$27.6 \pm 9.9 \%$ ) was observed in the intestine compared to the mesenteric lymph node $(1.7 \pm 1.6 \%)$ (Fig. 3B). A comparison of the expression of the CD8 $\alpha \beta$ heterodimer and the more unconventional $\mathrm{CD} 8 \alpha \alpha$ homodimer, showed that the vast majority of $\mathrm{CD}^{+} \mathrm{T}$ cells in the canine mesenteric lymph node and the intestinal epithelial layer expressed the CD $8 \alpha \beta$ heterodimer (small bowel: $49.7 \pm 8.6 \%$, large bowel: $59.3 \pm 12.2 \%$ ). In addition, we frequently observed relatively high numbers of 

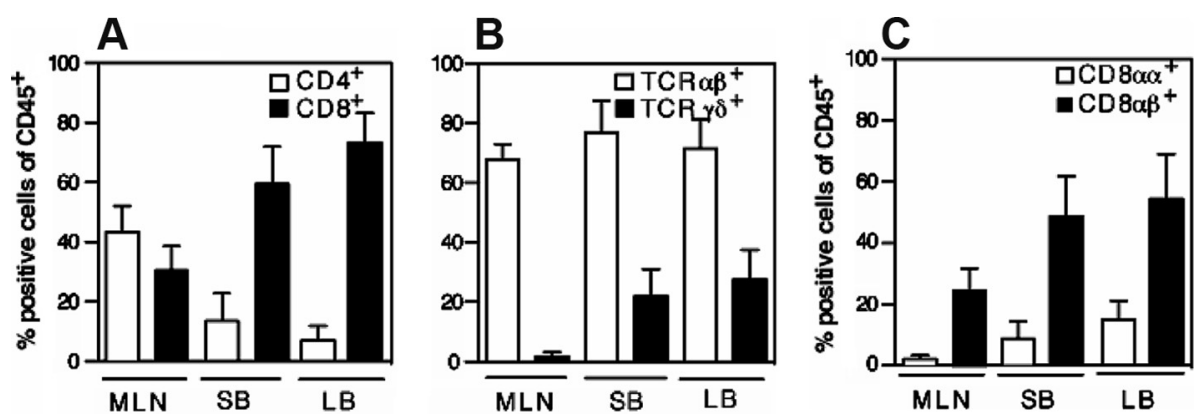

Figure 3. Lymphocyte subset composition of the canine intraepithelial compartment. Flow cytometric analysis of cell surface marker expression of mesenteric lymph node cells (MLN) and IEL from the small (SB) and large bowel (LB) from 15 healthy adult Beagle dogs showing analysis of $\mathrm{CD}^{+}$and $\mathrm{CD} 8^{+}$ lymphocyte subsets (A), TCR $\alpha \beta$ and TCR $\gamma \delta$ subsets (B), and CD8 $\alpha \beta^{+}$and $\mathrm{CD} 8 \alpha \alpha^{+}$subsets (C). Mean values $\pm \mathrm{SD}$ of $n=15$ are shown. Numbers were normalized to $\mathrm{CD} 45^{+}$cells.

unconventional T cells with the CD $8 \alpha \alpha$ homodimer in the intestinal epithelium (small bowel: $8.6 \pm 1.5 \%$, large bowel: $14.3 \pm 6.7 \%$ ), which were essentially absent in the mesenteric lymph node (Fig. 3C).

\subsection{Unconventional canine CD8 $\alpha \alpha$ IEL express the $\alpha \beta$ or $\gamma \delta \mathrm{T}$ cell receptor}

Using multi-parameter flow cytometric analysis, we assessed the TCR usage of CD $8 \alpha \beta^{+}$ and $\mathrm{CD} 8 \alpha \alpha^{+}$subsets. This revealed that the conventional $\mathrm{CD} 8 \alpha \beta^{+}$subsets predominantly express the TCR $\alpha \beta$ heterodimer, whereas within the unconventional $\mathrm{CD} 8 \alpha \alpha^{+}$subset, a high percentage of cells also expressed TCR $\gamma \delta$ (Fig. 4A). Detailed analysis of CD8 $\alpha \alpha^{+}$and CD $8 \alpha \beta^{+}$IEL from 4 dogs showed that TCR $\gamma \delta^{+}$ $\mathrm{T}$ cells are only a minor portion of $\mathrm{CD} 8 \alpha \beta^{+}$ IEL, whereas TCR $\gamma \delta$-expressing cells are concentrated in the CD8 $\alpha \alpha^{+}$IEL subset (Figs. 4B and 4C).

\subsection{Developmental changes in the IEL composition}

Significant differences could be found in the IEL subpopulations from neonatal and adult dogs. CD4 ${ }^{+}$TCR $\alpha \beta^{+}$IEL (Fig. 5A) and $\mathrm{CD}^{+} \mathrm{TCR}^{+} \beta^{+}$IEL (Fig. 5B) were only detected at low levels in newborn dogs but were present in much higher numbers in the small and large bowel of adult Beagle dogs. In contrast, substantial numbers of $\mathrm{CD}^{-} \mathrm{CD} 8^{-}$ TCR $\alpha \beta^{+}$IEL (Fig. 5C) and $\mathrm{CD}^{-} \mathrm{CD}^{-}$ TCR $\gamma \delta^{+}$IEL (Fig. 5D) were seen in the intestinal epithelial layer of neonates, but were present in much fewer numbers in adult dogs.

\subsection{Canine IEL respond poorly to mitogenic stimulation but display suppressive activities}

Analysis of proliferative responses revealed strong proliferation of mesenteric lymph node cells in response to mitogen (Fig. 6A) whereas IEL failed to do so. Co-culture experiments revealed that activation-induced proliferation of lymph node cells was suppressed in a dose-dependent fashion by IEL (Fig. 6B), demonstrating their regulatory potential. These findings were confirmed using highly purified lymphocytes (Fig. 6C). In contrast, purified intestinal epithelial cells failed to suppress activation-induced lymph node cell proliferation.

\subsection{Expression of immunoregulatory and inflammatory cytokines in canine IEL}

Quantitative PCR analysis of stimulationinduced expression of mRNA of the cytokines IFN $\gamma$, IL-10 and TGF $\beta$ revealed that both lymphocytes from the mesenteric lymph nodes and the IEL compartment expressed higher levels of 

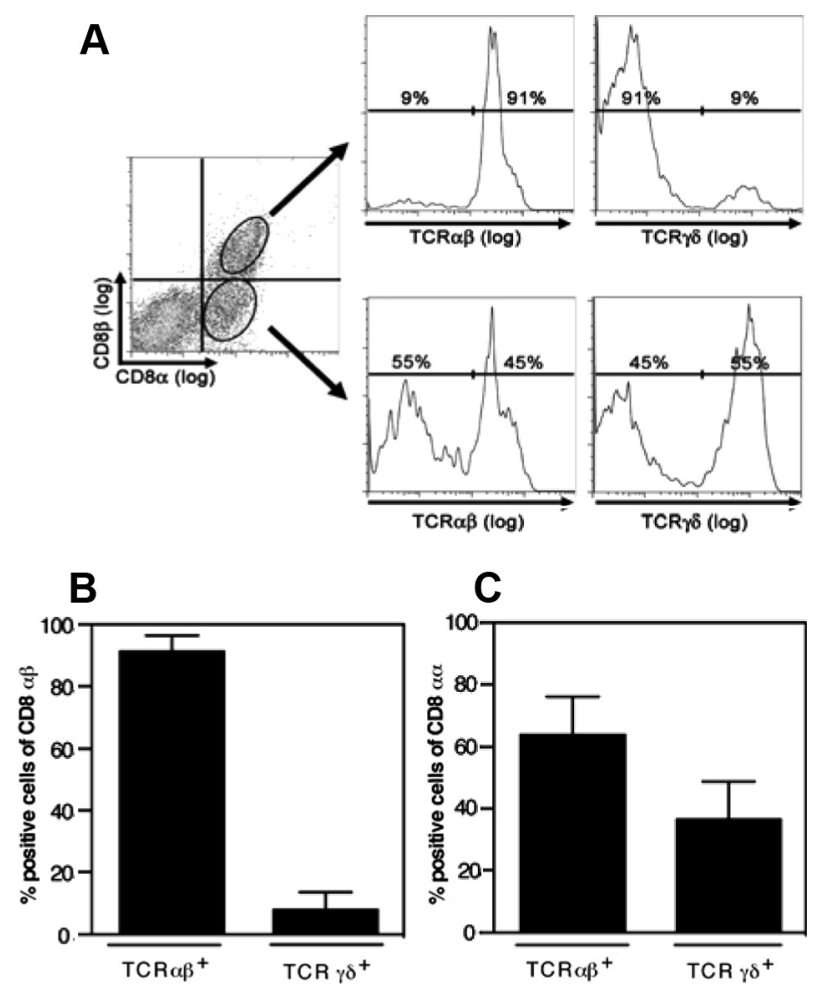

Figure 4. T cell receptor usage of canine intraepithelial CD8 $\alpha \alpha^{+}$and $\operatorname{CD} 8 \alpha \beta^{+} \mathrm{T}$ cells. T cell receptor usage of IEL from small bowel of healthy adult Beagle showing an example of CD8 $\alpha \beta^{+}$and CD8 $\alpha \alpha^{+}$subset detection and analysis of their TCR expression (TCR $\alpha \beta$ and TCR $\gamma \delta)(\mathrm{A})$, and analysis of TCR $\alpha \beta$ and TCR $\gamma \delta$ expression in the $\mathrm{CD} 8 \alpha \beta^{+}(\mathrm{B})$ and the $\mathrm{CD} 8 \alpha \alpha^{+}(\mathrm{C})$ subsets. Numbers were normalized to $\mathrm{CD} 8 \alpha \beta^{+}$ cells (B) and CD8 $\alpha \alpha^{+}$cells (C), respectively. Mean values \pm SD of $n=4$ are shown.

IFN $\gamma$ and IL-10 upon stimulation (Figs. 7A and 7B). There were no differences in the patterns of mRNA cytokine expression for both IFN $\gamma$ and IL-10 between IEL and mesenteric lymph node cells. Interestingly, both lymphocyte populations expressed lower levels of TGF $\beta$ upon stimulation (Fig. 7C).

\section{DISCUSSION}

The strategic location and effector functions of canine IEL suggest that they likely play a critical role in the maintenance of gut homoeostasis. Similarly, their development and composition is likely adapted to these needs. In both mice and man, intestinal IEL are generally subdivided into two major subpopulations based on their TCR usage and co-receptor expression [9]. The conventional (Type a) mucosal T cells comprise $\mathrm{CD}^{+}$and $\mathrm{CD} 8 \alpha \beta^{+}$ lymphocytes with the $\alpha \beta$ TCR, whereas the unconventional (Type b) subsets contain $\mathrm{CD} 8 \alpha \alpha^{+}$expressing TCR $\alpha \beta$ and $\mathrm{TCR} \gamma \delta$ as well as $\mathrm{CD} 4^{-} \mathrm{CD} 8^{-}$T cells. These IEL subpopulations do not only differ in their co-receptor and TCR usage but also show major functional and potentially developmental differences. Type a mucosal $\mathrm{T}$ cells have much in common with peripheral $\mathrm{CD}^{+}$or $\mathrm{CD} 8 \alpha \beta^{+}$cells, but also show some gut-specific regulatory functions. Thus, CD $8 \alpha \beta^{+}$TCR $\alpha \beta$ IEL may exert some cytolytic functions [4], whereas resident (non-infiltrating) $\mathrm{CD}^{+}$TCR $\alpha \beta$ cells often 


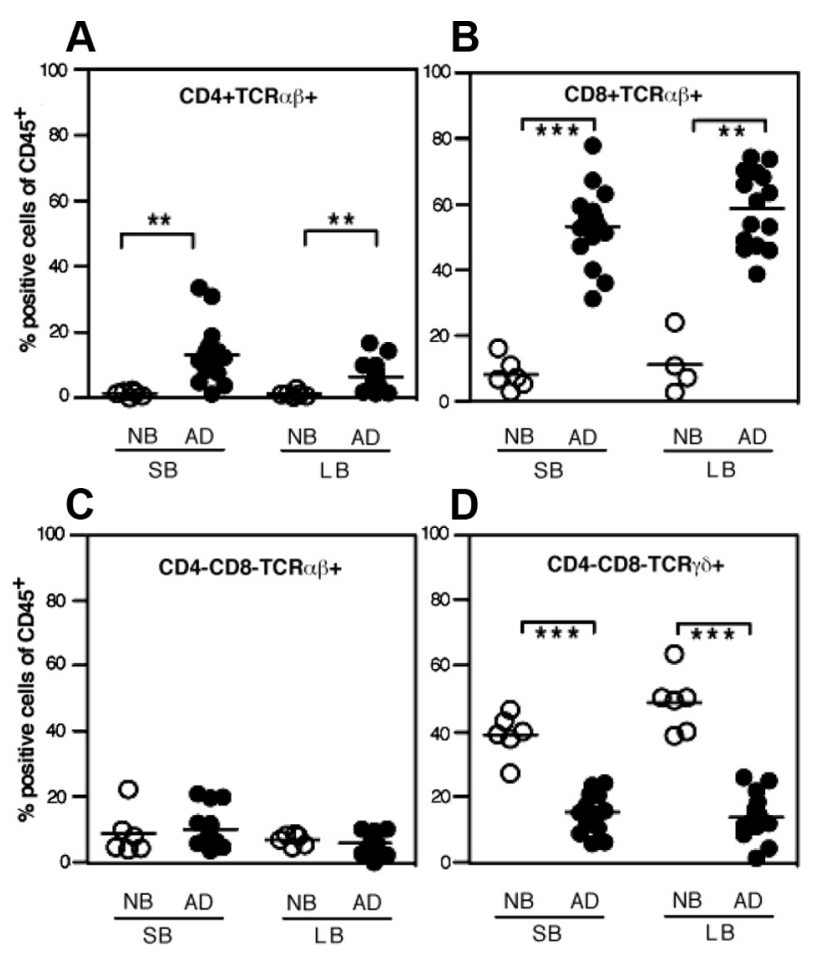

Figure 5. Differences in IEL subpopulations in the small and large bowel between newborn and adult dogs. Flow cytometrical characterization of leukocyte marker expression in IEL isolated from the small (SB) and large bowel (LB) in 15 healthy adult (AD) and 6 newborn (NB) Beagle dogs showing CD $4^{+}$TCR $\alpha \beta^{+}$IEL (A), $\mathrm{CD}^{+} \mathrm{TCR} \alpha \beta^{+}$IEL (B), $\mathrm{CD} 4^{-} \mathrm{CD} 8^{-} \mathrm{TCR} \alpha \beta^{+}$IEL (C), and $\mathrm{CD} 4^{-} \mathrm{CD} 8^{-} \mathrm{TCR} \gamma \delta^{+}$IEL (D). ** $p<0.005, * * * p \leq 0.0005$.

show a regulatory phenotype [3]. Type b $\mathrm{TCR} \gamma \delta$ cells likely play a dual role in the regulation of protective immune responses and the regulation of epithelial layer integrity via the release of keratinocyte growth factor [1]. Clearly, various IEL subsets play a critical role in the regulation of health and disease in the intestinal mucosa, and in the pathogenesis of IBD. Their abundance in the canine intestinal epithelium (Fig. 1) suggests that canine IEL may have a similarly important role in the regulation of intestinal immune homeostasis.

We carried out an in-depth phenotypical characterization of canine IEL using multiparameter flow cytometrical analyses. To date, only few flow cytometry-based studies on canine intestinal immune cells have been described $[18,21]$. The simultaneous detection of three parameters enabled us to demonstrate the differential TCR usage of $\mathrm{CD} 8 \alpha \beta^{+}$and CD $8 \alpha \alpha^{+}$IEL in the canine gut for the first time. As these distinct subsets have been previously shown to exert substantially different roles in intestinal immune responses in mice, their identification is likely relevant for the investigation of canine IBD [16]. Another major difference between previous investigations and the present study lies in the selection of study material [18, 21]; whilst previous studies used tissue biopsies harvested from various breeds using endoscopy, we studied full-thickness biopsies from a single canine breed in order to avoid inter-breed variability and to develop a mono-breed standard. These methodological differences may also explain some of the discrepancies between our results and those of previous studies. For 

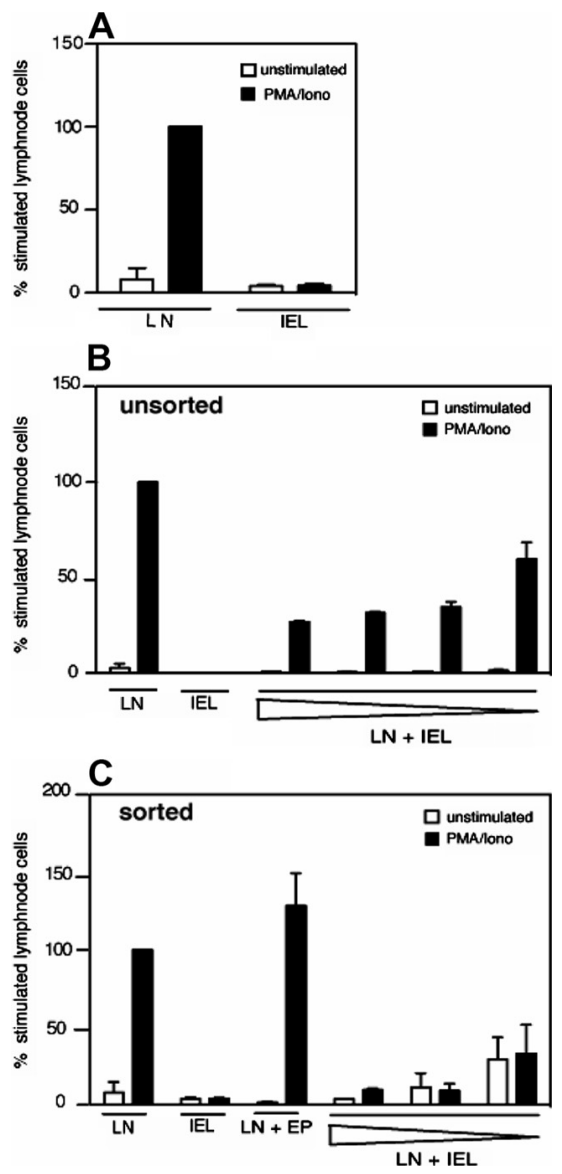

Figure 6. Canine IEL inhibit proliferation of mesenteric lymph node $T$ cells. The proliferative response of mesenteric lymph node cells (LN) and IEL from the small bowel of adult Beagle dogs, stimulated with medium control or PMA plus ionomycin and measured by $\left({ }^{3} \mathrm{H}\right)$ thymidine incorporation after $48 \mathrm{~h}$ (A). Mean values $\pm \mathrm{SD}$ of $n=5$ experiments are shown. (B) Unsorted mesenteric LN or IEL were either cultured alone with medium control or PMA plus ionomycin, or mesenteric lymph node cells were co-cultured with decreasing ratios of IEL. Mean values \pm SD of triplicates of typical experiments out of 5 are shown. The same experiment showing sorted IEL and intestinal epithelial cells (EP) as negative controls (C). Mean values $\pm \mathrm{SD}$ of triplicates of typical experiments are shown. Values (B and C) were normalized to the maximal proliferation of stimulated lymph node cells $(100 \%)$. example, our study revealed that $>95 \%$ of the cells in the lymphocyte gate were $\mathrm{CD} 45^{+}$and $\mathrm{CD}^{+}$with basically no $(<1 \%) \mathrm{CD} 21^{+} \mathrm{B}$ cells, indicating that IEL alone without contaminating lamina propria lymphocytes were assessed. This high percentage of T cells in the IEL compartment is well known in mice $[13,17]$ and was also suggested by previous immunohistochemical studies in dogs [7, 14]. However, these findings stand in contrast to those reported by Sonea and co-worker, in which only about $60 \%$ of the cells in the lymphocyte gate stained positive for CD45, among which only $50 \%$ were positive for CD3. As no density gradient centrifugation was performed in these studies, the low percentage of CD45 $5^{+}$cells in the lymphocyte gate may be due to contamination with (dying) epithelial cells. On the other hand, the absence of $\mathrm{CD}^{+}$cells may be explained by TCR downregulation of IEL due to prolonged incubation cycles during isolation. Clearly, our results demonstrate that canine IEL show a similar phenotypical pattern than that of mice and man [12, 13, 17], suggesting similar function in the regulation of local immune responses. Despite many similarities between canine and rodent IEL, we also observed quite disparate features of canine IEL, notably a high frequency of $\mathrm{CD} 4 / \mathrm{CD} 8$ double-negative IEL in adult dogs, which were even more pronounced in neonatal dogs. Also surprising was the high percentage of $\mathrm{CD}^{+}{ }^{+} \mathrm{TCR} \alpha \beta^{+} \mathrm{T}$ cells. In mice, an increase in $\mathrm{CD}^{+}{ }^{+} \mathrm{T}$ cells is usually indicative of ongoing immune responses and reflects the antigen-driven infiltration of peripheral $\mathrm{T}$ cells. Furthermore, the percentage of $\mathrm{CD}^{+} \mathrm{T}$ cells in the IEL compartment is generally higher in the large bowel due to the higher antigen load and bacterial colonization. In contrast, we observed that $\mathrm{CD} 4^{+} \mathrm{T}$ cells are found at higher frequency in the canine small bowel than in the large bowel. Moreover, this high percentage (up to $15 \%$ ) was observed in the absence of any signs of inflammation. Thus, canine CD4 ${ }^{+}$ IEL could represent a regulatory $T$ cell subset rather than pro-inflammatory $\mathrm{T}$ helper cells.

To the authors' knowledge, this study provides first-time evidence that canine IEL have a potent immunoregulatory potential. While 

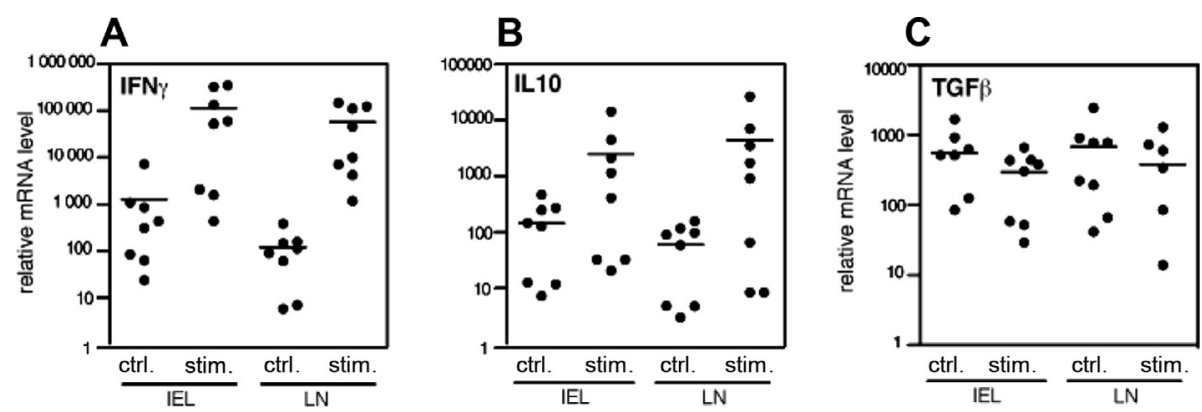

Figure 7. Cytokine expression profile of canine IEL. The expression of IFN $\gamma$ (A), IL-10 (B), and TGF $\beta$ (C) analyzed by real-time RT-PCR from mesenteric LN and IEL from small intestine of 8 adult Beagle dogs, isolated and stimulated for $6 \mathrm{~h}$ with medium control (ctrl.) or PMA plus ionomycin (stim.). Bars indicate mean values, symbols show values of individual samples.

mesenteric lymph node T cells strongly proliferated in response to mitogenic stimuli, IEL failed to do so as previously reported in other species [20]. However, co-culture of IEL with mesenteric lymph node cells, even at low ratios, substantially inhibited the proliferation of lymph node cells. This clearly indicates that IEL, or at least certain IEL subsets, lack conventional $\mathrm{T}$ cell effector functions and represent regulatory $\mathrm{T}$ cells. When analyzing the underlying mechanisms of IEL-based suppression of mesenteric lymph nodes, we failed to identify clear candidates. While there is good evidence that the immunoregulatory cytokines, IL-10 and TGF $\beta$, are critical effector molecule of IEL regulatory functions and oral tolerance induction in mice [11], we did not observe a clear expression pattern of these cytokines in regulatory IEL compared to mesenteric lymph node cells in dogs. This may have been due, in part, to the variety of $\mathrm{T}$ cell subsets with diverse expressions of these regulatory cytokines in both mesenteric lymph node and IEL preparations. For example, $\mathrm{CD}^{+}$regulatory T cells may also be present in mesenteric lymph node cells and pro-inflammatory $\mathrm{T}$ cells may also exist in the epithelial layer. Similarly, analysis of mRNA expression only partially reflects the actual protein production of these suppressive cytokines.

In conclusion, the present study represents the first multi-parameter characterization of intestinal IEL in a mono-breed population. This approach allowed the first description of $\mathrm{CD} 8 \alpha \alpha^{+} \mathrm{TCR} \alpha \beta^{+}$and $\mathrm{CD} 8 \alpha \alpha^{+} \mathrm{TCR} \gamma \delta^{+}$IEL subsets. Their abundance in the canine intestinal epithelial layer suggests an important role of these $T$ cell subsets in the maintenance of intestinal immune homeostasis. Our study also provides the first evidence that canine IEL have potent suppressive activities by suppressing mitogen-driven proliferation of peripheral $\mathrm{T}$ cells. The comparison of this model in a healthy mono-breed population of Beagle dogs with other breeds and with diseased dogs may advance the development of multi-parameter flow cytometry as a future diagnostic modality in canine IBD.

Acknowledgements. The authors thank Bernadette Wider for excellent help with cell sorting, and the members of the Brunner and Müller laboratory for intellectual and technical support. We also thank Judith Howard for English-language editing. Special thanks go to the Novartis Centre de Recherche Santé Animale (CRA, St. Aubin, Switzerland) and the RCC Ltd Laboratory Animal Service (Füllinsdorf, Switzerland) for their cooperation. This work was supported by grants from the Swiss National Science Foundation to T. Brunner and N. Corazza, and the Ettore and Valeria Rossi Foundation to N. Corazza.

\section{REFERENCES}

[1] Boismenu R., Havran W.L., Modulation of epithelial cell growth by intraepithelial gamma delta $\mathrm{T}$ cells, Science (1994) 266:1253-1255. 
[2] Brunner T., Arnold D., Wasem C., Herren S., Frutschi C., Regulation of cell death and survival in intestinal intraepithelial lymphocytes, Cell Death Differ. (2001) 8:706-714.

[3] Cheroutre H., IELs: enforcing law and order in the court of intestinal epithelium, Immunol. Rev. (2005) 206:114-131.

[4] Corazza N., Müller S., Brunner T., Kägi D., Mueller C., Differential contribution of Fas- and Perforin-mediated mechanisms of the cell-mediated cytotoxic activity of naive and in vivo-primed intestinal intraepithelial lymphocytes, J. Immunol. (2000) 164:398-403.

[5] Elwood C.M., Hamblin A.S., Batt R.M., Quantitative and qualitative immunohistochemistry of T cell subsets and MHC class II expression in the canine intestine, Vet. Immunol. Immunopathol. (1997) 58:195-207.

[6] German A.J., Hall E.J., Day M.J., Analysis of leucocyte subsets in the canine intestine, J. Comp. Pathol. (1999) 120:129-145.

[7] German A.J., Hall E.J., Moore P.F., Ringler D.J., Newman W., Day M.J., The distribution of lymphocytes expressing $\alpha \beta$ and $\gamma \delta$ T-cell receptors, and the expression of mucosal addressin cell adhesion molecule- 1 in the canine intestine, J. Comp. Pathol. (1999) 121:249-263.

[8] German A.J., Hall E.J., Day M.J., Immune cell populations within the duodenal mucosa of dogs with enteropathies, J. Vet. Intern. Med. (2001) 15:14-25.

[9] Hayday A., Theodoridis E., Ramsburg E., Shires J., Intraeptithelial lymphocytes: exploring the third way in immunology, Nat. Immunol. (2001) 2: 997-1003.

[10] Horio Y., Osawa S., Takagaki K., Hishida A., Furuta T., Ikuma M., Glutamine supplementation increases Th1-cytokine responses in murine intestinal intraepithelial lymphocytes, Cytokine (2008) 44: 92-95.

[11] Izcue A., Coombes J.L., Powrie F., Regulatory T cells suppress systemic and mucosal immune activation to control intestinal inflammation, Immunol. Rev. (2006) 212:256-271.

[12] Kakar S., Vandana N., Murray J., Dayharsh G.A., Burgart L.J., Significance of intraepithelial lymphocytosis in small bowel biopsy samples with normal mucosal architecture, Am. J. Gastroenterol. (2003) 98:2027-2033

[13] Klein J.R., Ontogeny of the Thy ${ }^{-1}, \mathrm{Lyt}^{2+}$ murine intestinal intraepithelial lymphocyte: characterization of a unique population of thymus independent cytotoxic effector cells in the intestinal mucosa, J. Exp. Med. (1986) 164:309-314.

[14] Kleinschmidt S., Meneses F., Nolte I., HewicherTrautwein M., Distribution of mast cell subtypes and immune cell populations in canine intestines: evidence for age-related decline in T cells and macrophages and increase of IgA-positive plasma cells, Res. Vet. Sci. (2008) 84:41-48

[15] Lefrançois L., Isolation of mouse small intestinal intraepithelial lymphocytes, in: Coligan J.E., Kruisbeek A.M., Marguelies D.H., Shevach E.M., Strober W. (Eds.), Current protocols in immunology, Wiley, New York, 1993, pp. 3.19.1-3.19.7.

[16] Müller S., Lory J., Corazza N., Griffiths G.M., Zgraggen K., Mazzuchelli L., et al., Activated CD4+ and CD8+ cytotoxic cells are present in increased numbers in the intestinal mucosa from patients with active inflammatory bowel disease, Am. J. Pathol. (1998) 152:261-268.

[17] Mosley R.L., Styre D., Klein J.R., CD4 ${ }^{+} \mathrm{CD} 8^{+}$ murine intestinal intraepithelial lymphocytes, Int. Immunol. (1990) 2:361-365.

[18] Sonea I.M., Jergens A., Sacco R.E., Niyo Y., Merten E., Kauffmann L.K., Moore P.F., Flow cytometric analysis of colonic and small intestinal mucosal lymphocytes obtained by endoscopic biopsies in the healthy dog, Vet. Immunol. Immunopathol. (2000) 77:103-119.

[19] Stokes C., Waly N., Mucosal defense along the gastrointestinal tract of cats and dogs, Vet. Res. (2006) 37:281-293.

[20] Sydora B.C., Mixter P.F., Holcombe H.R., Eghtesady P., Williams K., Amaal M.C., et al., Intestinal intraepithelial lymphocytes are activated and cytolytic but do not proliferate as well as other $\mathrm{T}$ cells in response to mitogenic signals, J. Immunol. (1993) 150:2179-2191.

[21] Zentek J., Hall E.J., German A., Haverson K., Bailey M., Rolfe V., et al., Morphology and immunopathology of the small and large intestine in dogs with nonspecific dietary sensitivity, J. Nutr. (2002) 132:1652-1654. 